\title{
Development of Granary Temperature and Humidity Prediction Model Based on RBF Neural Network
}

\author{
Qiu Weilin, Yang Siqing* \\ Information School, Hunan University of Humanities, Science and Technology, \\ Loudi 417000, China
}

\begin{abstract}
The traditional prediction methods are generally based on the assumption of linear model. At present, BP neural network and RBF neural network are usually adopted by people in prediction. Since BP neural network has the problem of local optimum and slow training speed, etc., in this paper the method of RBF neural network is adopted in modeling the granary temperature and humidity system, so as to represent the prediction model of granary temperature and humidity system and acquire a recursive prediction model. The experiments results showed that this model can represent the corresponding relationship between the input data vector and the output vector of granary.
\end{abstract}

Keywords: RBF neural network, Granary temperature and humidity, Prediction model

\section{Introduction}

Artificial neural network, which can be shorted for ANN, is a kind of intelligent learning algorithm developed in recent years with the development of artificial intelligence technology. Neural network is a kind of network system based on the basic principle of human brain neural network in biology, taking the theory of network topology as its basis, a complex information processing mechanism which can simulate human brain nervous system. This kind of network has the ability of parallel processing, high error tolerance, intelligence and self-learning ability, which has a high degree of nonlinearity and can carry out complex logic operations and nonlinear relations ${ }^{[1-3]}$. With the development of the research on neural network, great achievements have been made, for example, many practical problems have been solved in the fields of robotics, prediction and estimation, medicine and economy and so on, which has showed its better intelligent properties.

The development of artificial neural network has a long history, its development process is not so smooth. At the beginning of 1940s, the research development of neuroanatomy and neurophysiology as well as other subjects on neural network of human brains can make people have a certain understanding about the working principle of human brain neural networks. In 1969, M.Minsky and S. Papert issued a book called Perceptron, in this book they made a careful analysis on the the function and limitation of neural network system that took the perceptron as the representative, pointing out that the single perceptron can only be as a tool to distinguish line of classification, while multilayer perceptron can not give specific learning algorithm, which has no real value. Because of the efforts of Hopfierld as well as other people, coupled with BP (Back Propagation) proposed by D. E. Rumelhart and J.L.McClelland in 1986, who adopted error back-propagation training algorithm ${ }^{[4]}$. The neural 
network is the perfect solution to the multilayer perceptron learning algorithm for specific problems that Minsky and others thought it can not get, thus in all over the world it once again launched a study fad on artificial neural network. Today, the research of neural network is still a hot researching issue.

The traditional prediction methods are generally based on the assumption of linear model. At present, BP neural network and RBF neural network are usually adopted by people in prediction. Because BP neural network has the problem of local optimum and slow training speed, etc., therefore, in this paper the method of RBF neural network is adopted for modeling the granary temperature and humidity system, using RBF neural network for modeling the granary system is an approximation process, setting the temperature and humidity of granary system for training the neural network approach to the real through sample training, which can represent the corresponding relationship between the input data vector and the output vector of granary, so as to represent the prediction model of granary temperature and humidity system.

\section{RBF Neural Network}

\section{RBF Network Structure and Working Principle}

Radial basis function neural network (RBF network) is a typical three layer feedforward neural network, the first layer is input layer, the second layer is hidden layer and the third layer is output layer.

The basic idea of RBF neural network is: taking the activation function in each hidden layer, namely radial basis function as the "base" of space,such space can be called as the hidden layer space, the vector composed by the input data of the neural network can carry out a space transformation, which can map the low dimensional input data vector to a high dimensional implicit layer containing space, finally through the output of the hidden layer unit it can get the output that we need through the sum of linear weight, here the right is the adjustable parameter of the network ${ }^{[5-7]}$.

Among them, the radial basis function as the base of the hidden layer is a nonlinear function, its feature is partly distributed and symmetric about the center point, as for the hidden unit, after the center of the radial basis function is determined, the input data vector can be mapped to the hidden layer space. The radial basis function is usually selected in the following forms:

1) Gaussian function

$$
\Phi(t)=\mathrm{e}^{-\frac{t^{2}}{\sigma^{2}}}
$$

2) Reflected sigmoidal function

$$
\Phi(t)=\frac{1}{1+\mathrm{e}^{\frac{t^{2}}{\sigma^{2}}}}
$$

3) Inverse Multiquadric function

$$
\Phi(t)=\frac{1}{\left(t^{2}+\sigma^{2}\right)^{\alpha}}, \alpha>0
$$


In the formula (1-1) (1-3), it can be called as the expansion constant or width of the radial basis function. The smaller $\sigma$ is, the smaller the width of the corresponding function is, therefore the base function of the hidden layer has more choices. The activation function used in this paper is Gaussian function.

\section{Prediction Model of Granary Temperature and Humidity}

The granary temperature and humidity as the two most important factors can affect grain storage, therefore it is very necessary to control the granary temperature and humidity. But there is a big different with the conventional system, the coupling is existed between temperature and humidity in the granary, namely, controlling on the temperature will cause the change of humidity, humidity control will cause the temperature change, plus the inner apace of the granary is large, the change of temperature and humidity is a non linear, with strong coupling and large lag and slow time varying complex process. Besides, the influence of external environment, it is basically impossible to adopt the conventional modeling methods for the modeling and control. Therefore, in this paper it can adopt RBF neural network method for modeling the granary temperature and humidity system.

It is an approximation process for the granary system modeling by adopting RBF neural network training on the neural network so as to approach the real temperature and humidity of granary system through sample training, therefore, the accurate mathematical expression of prediction model of the temperature and humidity of granary system can not be given, only the following prediction model of the granary can be given:

$$
\hat{y}(k+1)=f[y(k) \ldots, y(k-\mathrm{N}+1), u(k) \ldots, u(k-\mathrm{M}+1), q(k) \ldots, q(k-L+1)]
$$

Among them, $\hat{y}(k+1)$ is prediction value of output at the next moment that the granary prediction model predicted, $y(k) \ldots, y(k-\mathrm{N}+1)$ respectively, is the actual output vector of granary system before the moment of $\mathrm{N} ; u(k) \ldots, u(k-\mathrm{M}+1)$ respectively, is the actual control output vector of granary system before the moment of $M ; q(k) \ldots, q(k-L+1)$ can represent temperature and humidity respectively of granary system before the moment of $L ; f$ can represent a simulating nonlinear function of granary. From the above we can see, through the actual output of $N$ granaries, actual control output of $M$, as well as the temperature and humidity data of $L$ granaries, we can predict the output of the next moment.

In addition, the above formula can also be extended to the $\mathrm{P}$ step ahead for prediction:

$$
\begin{array}{rl}
\hat{y}(k+P)=f & f \hat{y}(k+P-1) \ldots, \hat{y}(k+P-\mathrm{N}), u(k) \ldots, u(k+P-\mathrm{M}), \\
& q(k+P-1) \ldots, q(k+P-L)]
\end{array}
$$

In the above formula, when the moment of $k+P$ is predicted, the predicted value of $\hat{y}(k+i) \quad(\mathrm{i}=1,2 \ldots d-1)$ is used, as for the prediction value of the moment of $k$ as well as the moment before $k$ both can be replaced by the real value, namely: 


$$
\hat{y}(k+i)=y(k+i), i \leq 0
$$

It can roughly express the prediction model of granary temperature and humidity system through the above two equations, among them, $f$ is a recursive prediction model that got by RBF neural network, which can represent the corresponding relationship between the input data vector and the output vector of granary.

\section{Conclusion}

This paper introduces the development history of artificial neural network as well as some basic knowledge of RBF neural network. Then it makes a simple introduction about the approximately actual granary temperature and humidity system. Finally, a prediction model is given based on the granary temperature and humidity system.

\section{Reference}

[1] Hagan M T, Demuth H B, Beale M. Neural network design[M]. China Machine Press, 2002 .

[2] Ge S S, Harris C J. Adaptive Neural Network Control of Robotic Manipulators[J]. Proceedings of SPIE - The International Society for Optical Engineering, 2015, 4390:236-242.

[3] Zaremba W, Sutskever I, Vinyals O. Recurrent Neural Network Regularization[J]. Eprint Arxiv, 2015.

[4] Rumelhart D E, Hinton G E, Williams R J. Learning representations by back-propagating errors[J]. Nature, 1986, 323(6088):533-536.

[5] Qiao J, Han H. Optimal Structure Design for RBFNN Structure[J]. Acta Automatica Sinica,2010, 36(6):865-872.

[6] Fathi V, Montazer G A. An improvement in RBF learning algorithm based on PSO for real time applications[J]. Neurocomputing, 2013, 111(6):169-176.

[7] Han H G, Chen Q L, Qiao J F. An efficient self-organizing RBF neural network for water quality prediction[J]. Neural Networks the Official Journal of the International Neural Network Society, 2011, 24(7):717. 